\title{
Los conflictos en la tenencia forestal colectiva: Lecciones para Perú de un estudio comparativo
}

Anne M Larson, lliana Monterroso y Nicole Heise Vigil

\section{Mensajes clave}

- En comparación con Indonesia, Uganda y Nepal, la legislación peruana confiere un mandato débil para que los implementadores de la reforma de tenencia aborden los conflictos. Además, Perú tiene el menor número de funcionarios encargados de implementar la reforma que consideran que la atención de los conflictos es parte de sus responsabilidades.

- En las comunidades estudiadas, Perú reporta la mayor proporción de habitantes involucrados en conflictos forestales o por tierra, así como el porcentaje más alto de conflictos con actores externos a la comunidad y la menor proporción de conflictos resueltos.

- A pesar de la importancia legal del título de propiedad, la titulación colectiva no asegura por sí misma el fin de las disputas por tierras/bosques con personas externas. El Estado necesita defender los derechos de propiedad que ha reconocido.

- Perú debe mejorar su marco legal para el manejo de conflictos relacionados a disputas por tierras/ bosques tanto durante como luego de los procesos de formalización, tomando como base mecanismos estatales y consuetudinarios, comunitarios o alternativos.

\section{Introducción}

Las reformas de tenencia en tierras y bosques dirigidas a poblaciones tradicionales y otras comunidades que dependen del bosque han sido implementadas, entre otras razones, para reducir los conflictos por la tierra y los recursos. No obstante, las propias reformas pueden producir nuevos conflictos, por ejemplo, debido a la exclusión de miembros, cambios en los límites y contradicciones con las normas consuetudinarias (ver Coldham 2000; Fitzpatrick 2005; Gautam et al. 2014). Más aún, la titulación no necesariamente acaba con la competencia por la tierra o los recursos.

Este artículo se centra específicamente en las reformas de tenencia colectiva sobre las tierras forestales y los bosques. Aquí, las reformas se refieren a procesos de formalización que van desde titulación colectiva (en Perú) hasta regímenes de forestería social, forestería comunitaria o manejo forestal colaborativo (en Indonesia y Uganda). El conjunto de datos usado como referencia es complejo, las reformas y los contextos varían, y las conclusiones son matizadas. Sin embargo, los resultados señalan lecciones importantes para Perú, refuerzan nuestras observaciones desde el proyecto de investigación más amplio y sugieren importantes recomendaciones de políticas.
Este informe compara las perspectivas de los funcionarios de gobierno a cargo de la implementación de las reformas (en Perú, Nepal, Indonesia y Uganda) con las de las comunidades que han pasado por las reformas (en Perú, Indonesia y Uganda). También, examina el papel del gobierno en la gestión de conflictos, la naturaleza de los conflictos y la resolución de conflictos. La investigación no utilizó una definición predeterminada de conflicto sino más bien buscó entender las percepciones del concepto con relación a regímenes de tenencia colectiva mientras emergían en conversaciones con diferentes actores. Los consultados se refirieron a diferentes tipos de conflictos basados, en parte, en la naturaleza y escala de la pregunta, lo que es importante para interpretar los resultados y proponer soluciones. El informe explora la naturaleza y el alcance de la responsabilidad gubernamental para abordar los conflictos en los regímenes de tenencia colectiva.

Los resultados comparativos presentados aquí cuestionan la idea de que un título de propiedad es una forma segura de tenencia de la tierra, o que la titulación representa el punto final de un proceso para asegurar derechos. En Perú, se necesita prestar más atención a la resolución o transformación de conflictos en los regímenes de tenencia colectiva, tanto a través de las instituciones gubernamentales como consuetudinarias o alternativas para la resolución de disputas. 


\section{Métodos}

Esta investigación utiliza la información de un estudio comparativo con múltiples actores y múltiples métodos sobre las reformas de tenencia forestal desarrollado por CIFOR en siete países, de 2014 a 2017. ${ }^{1}$ El informe usa datos secundarios para examinar las leyes que abordan el papel del gobierno en la gestión de conflictos y dos fuentes principales de datos primarios de cuatro de los países estudiados. Primero, se hicieron 119 entrevistas con funcionarios del gobierno encargados de la implementación de reformas de tenencia de la tierra y bosques en Perú (32), Uganda (30), Nepal (29) e Indonesia (28). Estas personas se seleccionaron según el mapeo de pasos específicos y necesarios para la implementación de las reformas y, por lo tanto, representan a los funcionarios más importantes que intervienen en el proceso. Se preguntó a los encuestados gubernamentales sobre su responsabilidad en el manejo de conflictos, los tipos de conflictos que son llamados a atender y si los mecanismos de resolución son efectivos.

El segundo grupo de datos es a nivel de comunidad. La investigación incluyó 55 comunidades en Perú (22), Uganda (16) e Indonesia (17), que representan diferentes tipos de reformas, así como un pequeño números de comunidades con normas consuetudinarias (no reformadas). El conjunto de datos completo incluye 2,075 entrevistas de hogares realizadas a hombres y mujeres por separado. Se hizo un total de 136 entrevistas a informantes clave y 164 grupos focales con hombres y mujeres, también por separado. Algunos residentes de las comunidades en Indonesia y Uganda no participaron en las reformas. En este informe, se excluyeron las comunidades que no han experimentado reformas y los miembros de comunidades que no participaron en ellas para comparar solo a los participantes en las reformas. En resumen, el análisis de la encuesta incluye a 835 personas en Perú, 522 en Indonesia y 249 en Uganda. ${ }^{2}$ Se les preguntó si habían estado involucradas en conflictos durante el año anterior, el origen del conflicto, con quién era, si fue resuelto o no, y de ser así, quién lo resolvió. A nivel de comunidad, se pidió a los informantes clave y a los grupos focales que expliquen los conflictos tanto internos como externos (por ejemplo, empresas, gobierno, comunidades vecinas), así como la relación de los conflictos con las reformas de tenencia.

Los tipos de reformas estudiados (ver Tabla 1) varían ampliamente y están agrupados por "tipo de régimen", en base a tres de cuatro categorías utilizadas por la Iniciativa para los Derechos y Recursos (RRI 2018): Tierra estatal designada para uso de las comunidades, tierras en propiedad de las comunidades y tierra en propiedad de personas individuales. La muestra incluye una categoría adicional solo encontrada en Indonesia: Tierra estatal designada para uso de las empresas (las empresas negocian los derechos sobre la tierra con los pobladores). Se incluye solo un lugar con tierras privadas de propiedad individual (en Uganda) y un lugar donde algunos entrevistados habían participado en una reforma, aunque originalmente se había pensado que eran comunidades solamente con normas consuetudinarias y sin reformas (en Uganda). Para los fines de este informe, los resultados se han agrupado por país, pero no debe asumirse que representan hallazgos a nivel nacional, sino que resultan útiles para extraer pautas -especialmente lecciones para Perú- y para comparar las respuestas gubernamentales.

\section{Responsabilidad en el manejo de conflictos}

En los cuatro países, las leyes nacionales varían en el grado de responsabilidad que se da a los implementadores de las reformas de tenencia para el manejo de conflictos y en la provisión de mecanismos comunales. Perú parece tener el mandato gubernamental más débil y Uganda es el único país que tiene un mandato claro basado en la comunidad para la resolución de conflictos de tenencia.

\section{Tabla 1. Regímenes de tenencia incluidos en este análisis}

\begin{tabular}{|c|c|c|c|}
\hline \multirow{2}{*}{ Regímenes de tenencia } & \multicolumn{3}{|c|}{ Tipo de reforma (\# de comunidades) } \\
\hline & Uganda & Indonesia & Perú \\
\hline \multirow{2}{*}{$\begin{array}{l}\text { Tierra estatal designada para el } \\
\text { uso de las comunidades }\end{array}$} & \multirow{2}{*}{$\begin{array}{l}\text { Manejo forestal colaborativo } \\
(n=4)\end{array}$} & $\mathrm{HKm}^{*}$ (bosque comunal) $(\mathrm{n}=5)$ & \multirow{2}{*}{ - } \\
\hline & & $\mathrm{HTR}^{* *}$ (plantación forestal comunal) $(\mathrm{n}=2)$ & \\
\hline Tierra estatal usada por empresas & - & Kemitraan (asociación) $(n=4)$ & - \\
\hline \multirow{2}{*}{$\begin{array}{l}\text { Tierra de propiedad de las } \\
\text { comunidades }\end{array}$} & \multirow{2}{*}{$\begin{array}{l}\text { Bosque de la comunidad } \\
(n=4)\end{array}$} & \multirow{2}{*}{$\begin{array}{l}\text { Hutan Adat (bosque de uso } \\
\text { consuetudinario) }(n=1)\end{array}$} & $\begin{array}{l}\text { Comunidades nativas } \\
\text { tituladas }(n=17)\end{array}$ \\
\hline & & & $\begin{array}{l}\text { Comunidades campesinas } \\
\text { tituladas }(n=1)\end{array}$ \\
\hline $\begin{array}{l}\text { Tierra de propiedad de personas } \\
\text { individuales }\end{array}$ & $\begin{array}{l}\text { Asociación privada de } \\
\text { propietarios forestales }(n=4)\end{array}$ & - & - \\
\hline $\begin{array}{l}\text { Otras tierras de uso } \\
\text { consuetudinario reconocidas }\end{array}$ & $\begin{array}{l}\text { Tierras de uso consuetudinario } \\
\text { con algunas reformas }(n=4)\end{array}$ & - & - \\
\hline Total & 16 & 12 & 18 \\
\hline
\end{tabular}

1 Los otros países son: Colombia, la República Democrática del Congo y Kenia. La investigación a nivel de comunidad fue realizada solo en Indonesia, Perú y Uganda. La encuesta a los implementadores fue aplicada en estos tres países, así como en Nepal y Kenia, pero los resultados de Kenia no estuvieron disponibles al momento de elaborar este análisis.

2 Algunos resultados de cuatro comunidades que no han experimentado reformas (comunidades no tituladas) en Perú (171 encuestados) se incluyen para enriquecer el análisis sobre este país cuando resultan útiles. 
En Perú, la Ley de Descentralización de 2002 (Ley n²7867) pasó la responsabilidad de la titulación de tierras a los gobiernos regionales, sin embargo, esta ley no menciona el manejo de conflictos, y tampoco lo hacen las leyes de titulación de comunidades. Si las tierras de la comunidad están localizadas en áreas protegidas, la legislación sobre estas áreas (Art. 15 del Decreto Supremo AG N038/2001) ordena que sea el Comité de Gestión del área el que resuelva las disputas. En materia de mecanismos comunitarios de resolución de conflictos, la Constitución de 1993 reconoce la competencia de las comunidades indígenas sobre su territorios, de acuerdo a las normas consuetudinarias (siempre y cuando no se violen los derechos fundamentales de una persona), y la Ley de Comunidades Nativas de 1974 (22175, Art. 19) permite que la comunidad resuelva las disputas civiles menores. No obstante, en general, los procesos de titulación de tierras no incluyen un mandato específico sobre manejo de conflictos y lo que existe parece encontrarse disperso entre diversas organizaciones gubernamentales, sin lineamientos claros.

Por el contrario, las leyes en Indonesia reconocen explícitamente la existencia de conflictos en los procesos de formalización de tenencia de tierras, y sus mandatos son más claros. La Ley Forestal (Art. 74-76) aborda específicamente la solución de disputas forestales y señala que estas "pueden ser resueltas intra o extrajudicialmente, según la opción voluntaria de las partes en disputa" (Art. 74), aunque esto no aplica para actos criminales (RRI 2017). La Agencia Nacional de Tierras (BPN, por sus siglas en indonesio), que ahora está integrada con el Ministerio de Agricultura y de Ordenamiento del Territorio, es responsable del manejo de conflictos por tenencia de tierras, con leyes nacionales que definen las responsabilidades para la resolución de conflictos a nivel provincial, trans-provincial y trans-distrital (Ardiansyan et al. 2015). En este contexto, la legislación de Indonesia brinda un mandato claro para que el gobierno aborde los conflictos y para el uso de mecanismos alternativos (extrajudiciales), a pesar de que no especifica un mecanismo comunitario.

Uganda también reconoce claramente los conflictos, y el mandato para su resolución se otorga a una variedad de entidades:

"Actualmente, hay 5 mecanismos de resolución de disputas sobre tierras (...) establecidos en virtud de la Ley de Tierras de 1998 como parte de un esfuerzo para acercar los servicios relativos a la tierra y hacerlos más aceptables para los usuarios" (Uganda Land Alliance, 2014, p.185). Las Cortes de Consejos Locales, (LCCs, por sus siglas en inglés) son el nivel más bajo de cortes formalmente reconocidas en Uganda y pueden aplicar las leyes consuetudinarias o tradicionales, con base en la Constitución (Art. 129, 1995) y la Ley de Cortes de Consejos Locales. Estas operan como mecanismos de primera instancia para la mayoría de conflictos, incluyendo disputas sobre la tierra, y son económicamente accesibles. Adicionalmente, la Ley de Tierras reconoce los procedimientos consuetudinarios para la resolución de conflictos sobre tierras (Art. 5). Este enfoque hace que haya mecanismos de resolución de conflictos alternativos y tradicionales disponibles, a pesar de que también ha sido criticado por generar confusión y por "dar lugar a una acumulación de casos sin resolver" (Uganda Land Alliance, 2014, p. 185).
En Nepal, la Ley Forestal reconoce los conflictos que pueden surgir a causa de la demarcación de límites (por parte de la Oficina Forestal Distrital) y establece procedimientos claros para los reclamos (Art. 5-10) La Oficina Forestal Distrital gestiona esto, sin embargo, los miembros del comité pueden carecer de las habilidades necesarias (Shrestha 1998). La Ley Forestal autoriza que los Grupos de Usuarios Forestales (GUF) resuelvan conflictos locales hasta cierto punto, por ejemplo, les permite "imponer un castigo apropiado" (Art. 29) cuando se transgrede el plan de trabajo forestal de la comunidad. La autorización de los GUF "a menudo ha creado confusión en la resolución de conflictos de tenencia debido a que no se dan mayores directrices" (Yasmi et al. 2016). Adicionalmente, Heinen y Mehta (2000) argumentan que existen problemas al momento de articular responsabilidades entre diferentes instituciones gubernamentales.

Como parte de la investigación, se preguntó a los implementadores de las reformas si ellos o su oficina eran responsables de manejar o resolver los conflictos relacionados a la tenencia de la tierra. En general, la mitad respondió que "sí", que ellos son responsables, pero con diferencias importantes entre países (ver Figura 1). En Perú, solo 28\% dijo que sí. Es sorprendente de alguna manera que Indonesia y Uganda no registren valores más altos, debido a los mandatos legales con que cuentan. Sin embargo, esto puede deberse a que los mandatos más claros se dan acerca de la tierra, y la mayoría de reformas estudiadas involucran reformas forestales. Por el contrario, Nepal muestra un contraste substancial.

¿Es su responsabilidad o de su oficina manejar o resolver conflictos relacionados a tenencia?

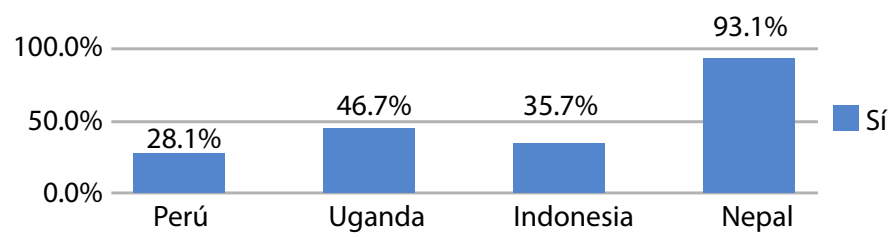

Figura 1. Percepción de los implementadores gubernamentales sobre su responsabilidad en el manejo de conflictos

\section{Comunidades involucradas en conflictos}

Se preguntó a los entrevistados en la encuesta a nivel de comunidad ${ }^{3}$ si habían estado involucrados en un conflicto forestal o de tierras durante el año anterior (ver Figura 2). Los mayores niveles de conflicto se encontraron en Perú y Uganda (cerca de 20\%), en comparación con solo $4 \%$ en Indonesia, y esta diferencia es significativa $(p<0.0001){ }^{4}$ En comunidades de Perú que no habían pasado por procesos de reforma (que no se muestran en el gráfico), solo 9\% de las personas estaban involucradas en conflictos. A pesar de que este país tiene uno de los niveles más altos de conflicto a nivel de comunidad, también tiene la proporción más baja de implementadores de las reformas que tienen en sus responsabilidades la resolución de conflictos.

3 Se excluye a Nepal porque la información no fue recolectada a nivel de comunidad.

4 Las diferencias son significativas según la prueba de chi-cuadrado de Pearson. Todas las pruebas estadísticas fueron realizadas con un nivel de confianza de 95\%. 


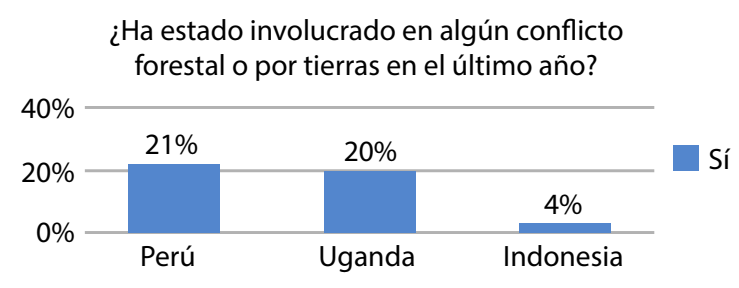

Figura 2. Miembros de la comunidad involucrados en un conflicto forestal o por tierras durante el último año

\section{Tipos de conflictos que los implementadores son llamados a abordar}

Se pidió a los implementadores de las reformas que respondieron que abordar conflictos es su responsabilidad, que describan los tipos principales de conflictos relacionados con tenencia que ellos son llamados a manejar o resolver. Las respuestas fueron abiertas y luego codificadas por el equipo de investigación (ver Tabla 2).

Las respuestas más comunes en Nepal y Uganda, y en segundo lugar en Perú, se relacionaban con problemas internos referidos a la gobernanza de los grupos de manejo forestal o de la comunidad. ${ }^{5}$ Los ejemplos incluyen: falta de consenso, de aplicación de la ley, de presupuesto y de transparencia, así como corrupción, captura por élites, distribución de beneficios y problemas con foráneos. En Perú, la respuesta más común se refirió a problemas institucionales, tales como falta de recursos, personal especializado o mecanismos de comunicación, funciones gubernamentales superpuestas y transparencia financiera. La falta de aplicación de la ley fue la tercera respuesta más común. En Indonesia, la respuesta más común fue límites de tierras poco claros/ superpuestos (segunda en Uganda), seguida de cerca por actividades de concesiones y empresas privadas. Los conflictos debido a restricciones sobre actividades locales de subsistencia fue la tercera respuesta más común en Uganda e Indonesia, y ha sido mencionada en otros estudios como un problema en Nepal (Shrestha 1998; Gautam et al. 2004).

\section{Tipos de conflictos desde la perspectiva de los miembros de la comunidad}

Se preguntó a los miembros de la comunidad que estuvieron involucrados en conflictos, con quién era el conflicto (ver Figura 3). En Indonesia y Uganda, más de la mitad fue con otros miembros de la comunidad. Esto sube hasta los tres cuartos si se suman los miembros familiares. En contraste, la mayor proporción en Perú fueron conflictos con actores externos y menos de un tercio con comuneros. Curiosamente, el 20\% decidió no responder la pregunta (posiblemente debido a lo delicado del tema). En comunidades que no experimentaron reformas en Perú, una proporción igual (31\%) reportó conflictos con actores externos y con miembros de la comunidad, y $25 \%$ prefirió no responder la pregunta; de manera notable, las comunidades que están tituladas tienen una porción más elevada de conflictos externos (41\% en comparación con 31\%).

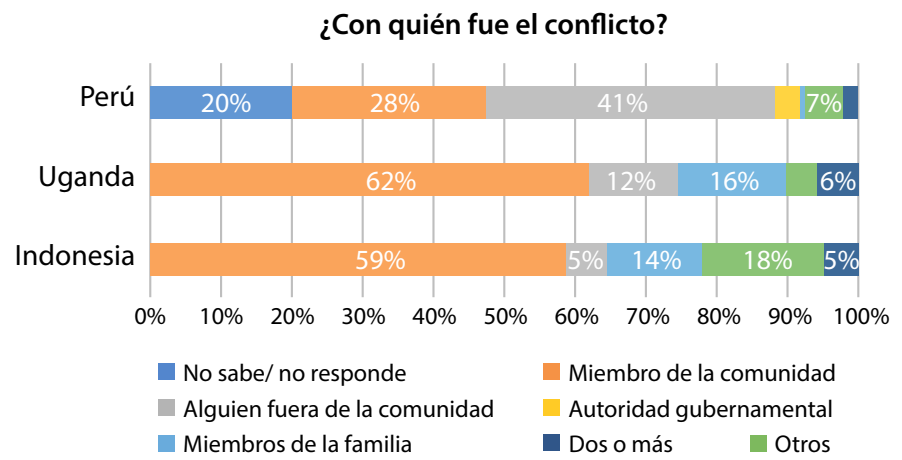

Figura 3. Reporte de los comunitarios encuestados sobre con quién fue el conflicto

\section{Tabla 2. Principales conflictos relacionados a tenencia que los implementadores han sido llamados a manejar o resolver, por país.}

\begin{tabular}{|c|c|c|c|c|}
\hline Tipo de conflicto & Indonesia & Nepal & Perú & Uganda \\
\hline Gobernanza del grupo de manejo forestal o de la comunidad & $0 \%$ & $32 \%$ & $20 \%$ & $26 \%$ \\
\hline Límites de tierras poco claros o superpuestos & $31 \%$ & $8 \%$ & $12 \%$ & $18 \%$ \\
\hline Debilidad institucional del gobierno o carencia de recursos (sin mención de la aplicación de la ley) & $8 \%$ & $6 \%$ & $28 \%$ & $4 \%$ \\
\hline Actividad de concesiones y empresas privadas & $27 \%$ & $0 \%$ & $0 \%$ & $4 \%$ \\
\hline Falta de regulación legal o de aplicación de la ley & $4 \%$ & $1 \%$ & $16 \%$ & $6 \%$ \\
\hline $\begin{array}{l}\text { Restricción a actividades económicas } \\
\text { locales o de subsistencia por ordenamiento territorial o políticas de conservación }\end{array}$ & $15 \%$ & $6 \%$ & $0 \%$ & $14 \%$ \\
\hline Distribución desigual de recursos naturales entre miembros de la comunidad o usuarios & $0 \%$ & $13 \%$ & $0 \%$ & $2 \%$ \\
\hline Usurpación/ invasión de tierras & $4 \%$ & $4 \%$ & $0 \%$ & $10 \%$ \\
\hline Tala ilegal, recolección ilegal, caza ilegal u otro tipo de extracción de recursos naturales & $4 \%$ & $7 \%$ & $8 \%$ & $6 \%$ \\
\hline Ataques de animales salvajes o desastres naturales & $0 \%$ & $8 \%$ & $0 \%$ & $4 \%$ \\
\hline Otros & $8 \%$ & $16 \%$ & $16 \%$ & $6 \%$ \\
\hline
\end{tabular}

5 Los grupos de manejo forestal son más comunes en Nepal y Uganda, mientras que la "comunidad" es con más frecuencia el punto de referencia en Perú. 
También se preguntó a los miembros de la comunidad involucrados en conflicto sobre el tema del mismo (Figura 4). Se permitió más de una respuesta. La diferencia entre países fue estadísticamente significativa $(p<0.005)$. La respuesta más común en los tres países fue sobre los límites, con una proporción abrumadora de esta respuesta en Uganda (71\%). El grupo de manejo forestal fue la segunda causa más común de conflictos en Indonesia (curiosamente, los implementadores del gobierno de Indonesia no lo mencionaron como un tipo de conflicto que ellos eran llamados a abordar). Se dio una variedad de otras respuestas, tales como derechos en disputa (entre individuos dentro de hogares) entre individuos y la comunidad y entre individuos y alguien de fuera de la comunidad) o usurpación e invasión de tierras. En las comunidades que no tuvieron reformas en Perú, los límites de tierras pocos claros o superpuestos también fueron la respuesta más común.

Los conflictos dentro de los hogares y la gobernanza de la comunidad empataron en el segundo lugar; pero no fueron tan importantes en las comunidades tituladas. (Ver también Recuadro 1).

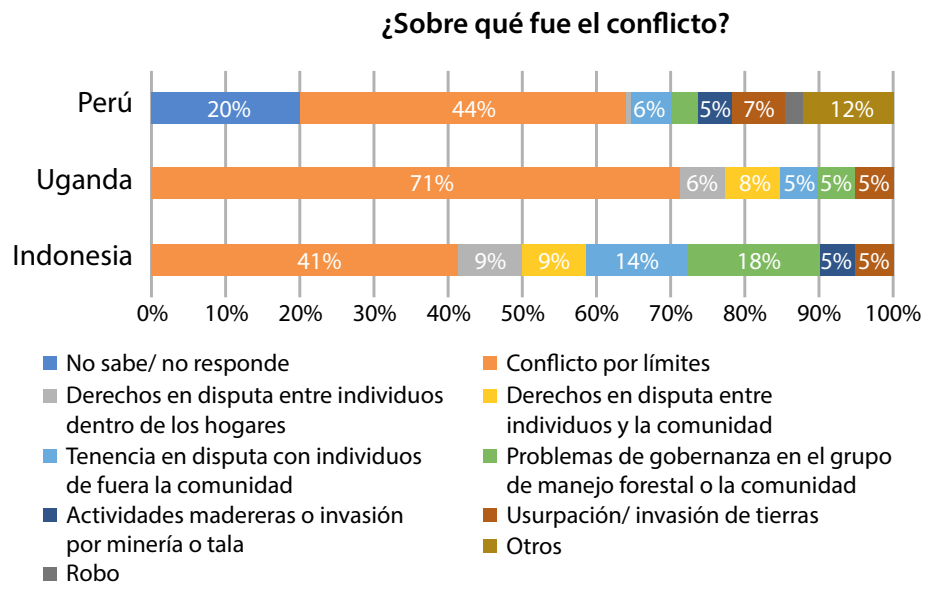

Figura 4. Reporte de los comunitarios encuestados sobre cuál fue el tema del conflicto

\section{La resolución de conflictos desde la perspectiva de las comunidades}

\begin{abstract}
Se preguntó a los miembros de la comunidad involucrados en conflictos durante el año anterior si estos se habían resuelto. La diferencia entre países es significativa ( $p<0.05)$, con casi todos los casos resueltos $(91 \%, n=22)$ en Indonesia, y 76\% en Uganda $(n=50)$, pero solo 44\% en Perú $(n=179)$. Además, es importante notar que el número total de conflictos en Perú era mucho más alto. Las comunidades que no han experimentado reformas en Peru también informaron que $44 \%$ de los casos fueron resueltos $(n=78)$.
\end{abstract}

\begin{abstract}
También se preguntó a los pobladores sobre quién había resuelto el conflicto (Figura 5). No hubo diferencias significativas entre los países: las autoridades internas de la comunidad, elegidas o no, resolvieron la gran mayoría de conflictos. Las autoridades elegidas solucionaron la mayoría de casos en Uganda y Perú, mientras que otros líderes de la comunidad resolvieron la proporción más alta en Indonesia. En Perú e Indonesia, pero no en Uganda, otros comités locales (cooperativas, comités de manejo de conflictos) resolvieron un gran número de casos. Las autoridades gubernamentales resolvieron un número importante de conflictos en las comunidades no tituladas en Perú (29\%), pero los líderes elegidos ahí resolvieron el 57\%. En Uganda, el gobierno resolvió el $13 \%$ de los conflictos. Los procesos judiciales/litigios solo fueron importantes en Uganda para el 8\% de los consultados (en comparación con el 1\% en Perú). Esto puede ser debido a que las Cortes de Consejos Locales en Uganda hacen que los procesos judiciales sean más accesibles para los pobladores en comparación con otros países.
\end{abstract}

Estos resultados demuestran la importancia de los mecanismos de resolución de conflictos a nivel de la comunidad. No obstante, debido al alto número de conflictos en Perú y a la gran proporción que queda sin resolver, tales mecanismos pueden ser insuficientes. Esto puede deberse, en parte, a que una gran porción de conflictos fueron en realidad con actores externos a la comunidad.

\section{Recuadro 1. Conflictos a nivel de comunidad y reformas de tenencia}

Se pidió a los informantes clave y a los grupos focales (GF) que explicaran los conflictos internos y externos en sus comunidades. En Indonesia, la mayoría de GF e informantes clave reportó pocos conflictos. Algunos mencionaron específicamente que la reforma funcionaba como un mecanismo de resolución de conflictos y ayudaba a las comunidades "ilegales" a obtener acceso legal a la tierra. Solo uno de los informantes clave expresó que la reforma era causa de conflictos porque no cumplía con las expectativas de la comunidad. En las discusiones de los GF, se mencionaron conflictos con las empresas privadas, pero no fueron vinculados con el proceso de reforma.

En Uganda, la mayoría de informantes clave y participantes de los GF no mencionó la reforma como causa de conflicto, pero sí señaló los conflictos con autoridades del gobierno. Los informantes clave reportaron conflictos con el gobierno debido a la falta de apoyo, restricciones de las políticas de conservación, desconfianza sobre derechos de tenencia de tierras (tierra/propiedad que es arrebatada) y corrupción.

En Perú, por el contrario, muchos de los informantes clave y GF mencionaron la reforma misma como causa de conflicto. Por ejemplo, en una comunidad, un informante clave señaló que durante el proceso de titulación su comunidad tuvo que ceder parte de su territorio ancestral. En otras comunidades, los informantes clave y GF dijeron que se habían establecido límites nuevos y poco claros (debido a procedimientos de consulta inadecuados y superposiciones) y que la tierra fue entregada a foráneos durante la titulación. Algunos afirmaron que se crearon problemas internos porque el proceso de titulación requería el establecimiento de una junta directiva que luego estuvo involucrada en manejo financiero corrupto. Tanto en las comunidades tituladas (con reforma), como no tituladas (sin reforma), los consultados comunicaron problemas de usurpación de tierras con comunidades vecinas, madereros ilegales, mineros ilegales y otros. 


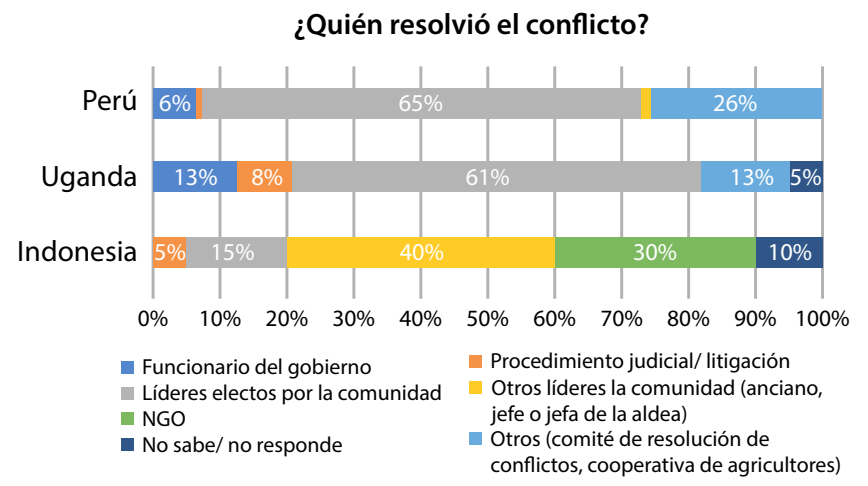

Figura 5. Reporte de los comunitarios encuestados sobre quién resolvió el conflicto

\section{La resolución de conflictos desde la perspectiva de los implementadores}

También se preguntó a los implementadores de la reforma con responsabilidad en el manejo de conflictos, cuán efectivos eran los mecanismos de resolución de conflictos (Figura 6). En todos los países, la mayoría de los consultados señaló que los mecanismos eran efectivos (muy efectivos/efectivos/algo efectivos). Sin embargo, aunque Perú tenía la mayor proporción que afirmaba que estos eran muy efectivos/efectivos, un tercio dijo que los mecanismos eran inefectivos. Este fue también el número más alto entre todos los países estudiados.

¿Cuán efectivos fueron los mecanismos de resolución de conflictos para resolver los conflictos?

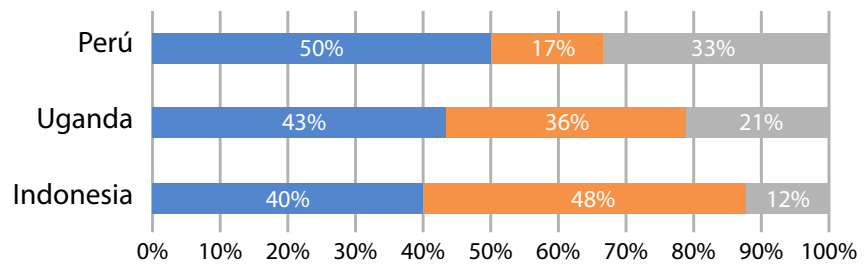

- Muy efectivos/ efectivos algo efectivos Inefectivos

Figura 6. Opinión de los implementadores gubernamentales sobre la efectividad de los mecanismos de resolución de conflictos

\section{Discusión: Lecciones para Perú}

Perú tiene el marco legal más débil para manejo de conflictos, con mandatos poco claros para el gobierno, especialmente en la titulación de comunidades nativas. No es de sorprender entonces que Perú tenga el porcentaje más bajo de funcionarios oficiales que señala que tienen responsabilidad en el manejo de conflictos y el porcentaje más alto que afirma que los mecanismos de resolución de conflictos son inefectivos (aunque en conjunto, más son efectivos que inefectivos).

La falta de mandato genera preocupación ante los hallazgos a nivel de comunidad: Perú tiene el porcentaje más alto de miembros de la comunidad que reporta conflictos durante el año anterior y el porcentaje más bajo de resolución. Si el problema fuera interno a las comunidades, esto podría sugerir la necesidad de reforzar los mecanismos comunales de resolución de conflictos. Sin embargo, Perú tiene también una porción muy elevada de miembros de las comunidades (41\%) que reporta conflictos con personas externas a sus comunidades. En comparación, Uganda parece tener los mecanismos alternativos para resolución de disputas más sólidos. En la práctica, los resultados sugieren que todas las comunidades estudiadas en Uganda, Perú e Indonesia dependen en gran medida de los líderes y comités locales para la resolución de conflictos.

Aunque el título sobre la tierra es la forma más sólida de derechos legales de tenencia, los resultados muestran que la titulación por sí misma está lejos de ser suficiente para abordar los conflictos. La comparación de resultados tanto en comunidades con titulación como sin ella en Perú, refuerzan este hallazgo: el porcentaje de pobladores de comunidades tituladas que tuvieron conflictos durante el año anterior fue más del doble que en las comunidades no tituladas. Lo más preocupante es que los conflictos con actores externos fueron más en las comunidades tituladas que las no tituladas (41\% versus $31 \%$ ). Esto sugiere que la definición legal de límites no ha detenido la intrusión. En realidad en Perú los miembros de comunidades y los líderes describieron el proceso de titulación en sí mismo como una causa de conflicto.

\section{¿De qué tipos de conflicto estamos hablando?}

La definición de conflicto se dejó abierta a la interpretación de los entrevistados y se obtuvo diferentes respuestas. Se preguntó a los funcionarios de gobierno que implementaban las reformas de tenencia qué tipos de conflictos eran llamados a manejar o resolver; a la vez se preguntó a los pobladores, hombres y mujeres, sobre conflictos específicos relacionados a tierras o bosques en los que hubieran tomado parte durante el año anterior. En el contexto de las reformas colectivas, los primeros tienen un enfoque claro a nivel de comunidad -o de grupo-, mientras los últimos se refieren al individuo. Se preguntó también a los informantes clave y grupos focales sobre conflictos a nivel comunal. Las repuestas de los funcionarios gubernamentales se basaron en su trabajo y en el nivel nacional, mientras que las comunidades estudiadas solo representan una proporción muy pequeña del total. Los resultados fueron bastante consistentes para Indonesia y Uganda, pero menos para Perú.

La respuesta más común de los oficiales del gobierno y los miembros de las comunidades en los tres países fue que los conflictos se referían a los límites. En Indonesia, las comunidades estudiadas tenían, en general, niveles muy bajos de conflicto, pero los límites eran la causa más común de conflicto, según el gobierno y los miembros de las comunidades. Ambos se refirieron además a conflictos con empresas privadas. En Uganda, la respuesta principal de los entrevistados gubernamentales hacía referencia a conflictos con grupos de manejo forestal, mientras que, como en respuesta, los informantes clave y los grupos focales aludían a conflictos con las autoridades gubernamentales. Los conflictos por límites fueron la respuesta principal de los comunitarios y el segundo conflicto más importante mencionado por el gobierno.

Las respuestas en Perú fueron más variadas y ayudan a comprender la naturaleza del problema y las formas de resolverlo. Primero, los tres tipos de grupos consultados se refirieron a los límites como un problema, pero este tema se encontraba bastante abajo en la lista del gobierno. Esto ilustra que el gobierno es raramente convocado para abordar el problema más importante que enfrentan los comunitarios. Esto sería apropiado si los conflictos por límites se dieran entre individuos dentro de una comunidad titulada, pero, como se señaló antes, los resultados sugieren que no es así. 
En segundo lugar, los funcionarios gubernamentales reportaron que el "conflicto" más importante era la debilidad del gobierno en sí (por ejemplo, personal, comunicación, presupuesto), seguida por la gobernanza de la comunidad y luego, por la aplicación de la ley. El primero y tercero de estos se refieren a deficiencias del gobierno (dos temas escasamente mencionados en los otros países).

En tercer lugar, tal como los implementadores gubernamentales, los grupos focales e informantes clave a nivel de comunidad hicieron referencia a problemas con la gobernanza comunal, pero, para ellos, esta estaba directamente asociada con el proceso de titulación. Algunos argumentaron que los nuevos órganos de liderazgo de la comunidad dieron lugar a la corrupción (sin embargo, en otras comunidades, las personas hablaron bien de los órganos de gobierno, por ejemplo, en la reducción de conflictos con colonos externos). El proceso de titulación también fue culpado por la pérdida de territorio y por hacer los límites más confusos, en lugar de aclararlos. Entre otras cosas, estas respuestas demuestran desconfianza mutua entre las comunidades y el gobierno.

En cuarto lugar, invasiones de varias formas realizadas por personas externas fueron problemas clave para los individuos y los miembros de la comunidad, pero no fueron mencionadas por los implementadores gubernamentales como tales. Si bien algunos de estos conflictos pueden tratarse de lo que fue referido por el gobierno como problemas con la aplicación de la ley, las grandes diferencias sugieren una mayor desconexión entre las necesidades de las comunidades indígenas y el mandato gubernamental para encontrar soluciones.

\section{¿Quién debería resolver los (y cuáles) conflictos?}

Se necesitan mecanismos y enfoques adecuados para el manejo y transformación de conflictos según la naturaleza del conflicto en cuestión. Idealmente, las comunidades indígenas deberían resolver sus propios problemas siempre que sea posible. Sin embargo, no todos los conflictos relacionados a la tenencia de tierras o forestal son internos en las comunidades. Algunos conflictos externos requieren apoyo desde más allá del nivel comunal para su resolución. Esto es cierto particularmente si el conflicto en cuestión está relacionado a la defensa del derecho de propiedad de la comunidad.

Por definición, un título de propiedad tiene sentido precisamente porque está respaldado por el Estado que lo emite. Los problemas de límites entre las comunidades, la usurpación por parte de vecinos o el robo de los recursos de la comunidad por foráneos son de competencia estatal. Esto no quiere decir que se necesita la intervención del Estado en cada uno de los casos, sino que es su responsabilidad establecer las instituciones para defender y proteger los títulos emitidos, así como los derechos de los ciudadanos indígenas del país. Esto incluye crear mecanismos accesibles e inclusivos para la resolución y transformación de conflictos.

\section{Gobierno y manejo de conflictos en Perú}

Los problemas de coordinación interna y funciones gubernamentales que se superponen pueden causar confusión y agravar los conflictos (Baranyi y Weitzner 2006; Herrera y Guglielma da Passano 2006). En Perú, Huamaní et al. (2012) han argumentado que las instituciones de manejo de conflictos padecen especialmente de desarticulación, y que existe escasez de especialistas del gobierno para manejar conflictos. Un reporte gubernamental encontró que la respuesta inadecuada de las entidades públicas fue una de las causas principales de conflicto en la región amazónica (PCM 2018), y académicos en Perú concuerdan con que el alto nivel de conflictos se debe en gran parte a la limitada capacidad institucional para el manejo de conflictos (Tanaka 2011; Huamaní et al. 2012; Orihuela y Paredes 2017).

Perú ha desarrollado un sistema de manejo de conflictos para abordar problemas con industrias extractivas y una regulación socioambiental (Huamani et al. 2012). Sin embargo, este sistema es desorganizado y centrado en crisis, una señal probable de problemas más profundos. Merino (2015) sostiene que los conflictos en Perú son un asunto de ontologías políticas divergentes, donde el Estado ve la Amazonía como un lugar para la explotación de recursos naturales, mientras ignora otras interacciones con la naturaleza. Se necesita un nuevo marco legal holístico, con un enfoque intercultural que incluya las perspectivas de los pueblos indígenas.

\section{Conclusiones y recomendaciones}

Esta investigación demuestra que la titulación de comunidades por sí misma es insuficiente para la defensa del territorio: la entrega de un título no es "el fin" del proceso para asegurar los derechos de las comunidades indígenas, ${ }^{6}$ ni tampoco el gobierno queda libre de responsabilidades una vez que el título es concedido.

Como señalan las Directrices Voluntarias sobre la Gobernanza Responsable de la Tenencia de la Tierra, la Pesca y los Bosques (VGGT, por sus siglas en inglés), los conflictos por tenencia de tierras necesitan contar con acceso efectivo a la justicia y a mecanismos de resolución de conflictos. El Estado es responsable de proteger a la población indígena en cualquier transacción con privados y de respaldar la capacitación para que cualquier proceso de consulta sea desarrollado apropiadamente. Esto debe combinarse con la lucha contra la corrupción.

En Uganda, las instituciones de resolución de conflictos subrayan la importancia de involucrar a las autoridades locales en sistemas formales de resolución de conflictos, algo que debería tomarse en cuenta en la estructuración de un marco legal para el manejo de conflictos en Perú. Baranyi y Weitzner (2006) mencionan además que la capacitación es necesaria para todas las partes y para facilitar la resolución de los conflictos relacionados a la tierra.

En resumen, los marcos legales para el manejo de conflictos necesitan reconocer a las autoridades locales y consuetudinarias en los procesos de resolución de disputas, establecer procedimientos claros para los conflictos de tenencia de tierras/bosques, e instaurar mandatos de resolución de conflictos para los funcionarios gubernamentales. El conflicto debe abordarse no solo durante el proceso de formalización, sino también después de que la formalización se haya completado.

6 Ni los medios de vida, que también merecen el apoyo del Estado. 


\section{Agradecimientos}

El Estudio Comparativo Global sobre Reformas de Tenencia en Tierras Forestales de CIFOR es financiado por la Comisión Europea y el Fondo para el Medio Ambiente Mundial (FMAM) y cuenta con el respaldo técnico del Fondo Internacional para el Desarrollo Agrícola (FIDA) y la Organización de las Naciones Unidas para la Alimentación y la Agricultura (FAO). También agradecemos a Safia Aggarwal, Peter Veit, Alain Frechette y Sondra Wentzel por sus útiles revisiones y comentarios y a Karin Rivera por su apoyo en el análisis estadístico. Este estudio forma parte del Programa sobre Políticas, Instituciones y Mercados (PIM), dirigido por el Instituto Internacional de Investigación sobre Políticas Alimentarias (IFPRI); y el Programa de Investigación del CGIAR sobre Bosques, Árboles y Agroforestería (FTA), liderado por CIFOR. Las opiniones aquí expresadas pertenecen a las autoras y no reflejan necesariamente los puntos de vista de CIFOR, CGIAR o los patrocinadores financieros.

\section{Referencias}

Ardiansyah, F., Marthen, A.A. y Amalia, N. 2015. Forest and Land-use Governance in a Decentralized Indonesia: A Legal and Policy Review. Occasional Paper No. 132. Bogor, Indonesia: CIFOR.

Baranyi, S. y Weitzner, V. 2006. Transforming Land-related Conflict: Policy, Practice and Possibilities. Ottawa y Roma: North-South Institute.

Coldham, S. 2000. Land reform and customary rights: the case of Uganda. Journal of African Law 1: 65-77.

Fitzpatrick, D. 2005. 'Best practice' options for the legal recognition of customary tenure. Development \& Change 36(3): 449-475.

Gautam, A. Shivakoti, G. y Webb, E. 2004. A review of forest policies, institutions, and changes in the resource condition in Nepal. The International Forestry Review 2(6): 136-148.

Heinen, J. y Mehta, J.N. 2000. Emerging issues in legal and procedural aspects of buffer zone management with case studies from Nepal. The Journal of Environment \& Development 9: 45-67.
Herrera, A. y Guglielma da Passano, M. 2006. Land Tenure Alternative Conflict Management. Roma: FAO.

Huamaní, G., Macassi, S., Alegría, J., y Rojas, T. 2012. Hacia una mejor gestión de los conflictos socioambientales en el Perú. Lima: Consorcio de Investigación Económica y Social.

Merino, R. 2015. The politics of extractive governance: Indigenous peoples and socio-environmental conflicts. The Extractive Industries and Society 1 (2): 85-92.

Orihuela, J. y Paredes, M. 2017. Fragmented layering: building a green state for mining in Peru. En Dargent, E., Orihuela, J., Paredes, M., y Ulfe, M. (eds.) Re source Booms and Institutional Pathways. Cham: Palgrave Macmillan

Presidencia del Consejo de Ministros de la República del Perú (PCM). 2018. Reporte mensual agosto 2018. Revista Willagniki, no. 4. Lima, Perú: PCM.

Shrestha, K.B. 1998. Community Forestry and Conflicts in Nepal. En $\mathrm{FAO}$, Integrating conflict management considerations into national policy frameworks: Proceedings, Antalya, Turquía, Oct. 10-13, 1997 (pp. 283-290). Roma: FAO.

Tanaka, M. 2011 ¿Por qué el Estado no responde adecuadamente a los conflictos sociales? Y ¿qué hacer al respecto? Lima, Perú: IEP (Instituto de Estudios Peruanos), CIES (Consorcio de Investigación Económica y Social).

Rights and Resources Initiative (RRI). 2017. Power and Potential: A Comparative Analysis of National Laws and Regulations Concerning Women's Rights to Community Forests. Washington, DC: RRI.

Rights and Resources Initiative (RRI). 2018. At a Crossroads. Consequential trends in recognition of community-based forest tenure from 2002-2017. Washington, DC: RRI.

Obaikol, E. 2014. Draft Final Report of the Implementation of The Land Governance Assessment Framework in Uganda. Washington, DC: World Bank Group.

Yasmi, Y., Anshari, G., Komarudin, H., y Alqardi, S. 2016. Forest tenure policies in Nepal: Status, gaps and way forward. Roma: FAO Regional Office for Asia and the Pacific.

\begin{tabular}{|c|c|c|}
\hline$\underbrace{b j}_{\text {CGIAR }}$ & $\begin{array}{l}\text { RESEARCH } \\
\text { PROGRAM ON } \\
\text { Policies, } \\
\text { Institutions, } \\
\text { and Markets } \\
\quad \text { Led by IFPRI }\end{array}$ & $\begin{array}{l}\text { El programa sobre Políticas, Instituciones y Mercados (PIM) del CGIAR, lidera investigación orientada a la acción para informar a los } \\
\text { tomadores de decisión con la evidencia necesaria para desarrollar políticas alimentarias y agrícolas que puedan servir de mejor } \\
\text { manera los intereses de los productores y consumidores, tanto hombres y mujeres. PIM combina los recursos de los centros de } \\
\text { investigación del CGIAR, así como numerosos socios a nivel internacional, regional y nacional. El programa es liderado por el } \\
\text { Instituto Internacional de Investigación sobre Políticas Alimentarias (IFPRI, por sus siglas en inglés). www.pim.cgiar.org }\end{array}$ \\
\hline
\end{tabular}

\begin{tabular}{|l|l} 
CGJ & $\begin{array}{l}\text { RESEARCH } \\
\text { PROGRAM ON }\end{array}$ \\
Forests, Trees and & Agroforestry
\end{tabular}

Esta investigación fue realizada por CIFOR como parte del Programa de Investigación de CGIAR sobre Bosques, Árboles y Agroforestería (FTA). El FTA es el programa de investigación para el desarrollo más grande del mundo, dedicado a mejorar el papel de bosques, árboles y la agroforestería para el desarrollo sostenible, la seguridad alimentaria, y frente al cambio climático. CIFOR dirige el programa FTA en asociación con Bioversity International, CATIE, CIRAD, ICRAF, INBAR y TBI. La investigación del Programa FTA cuenta con el apoyo del Fondo Fiduciario del CGIAR: cgiar.org/funders
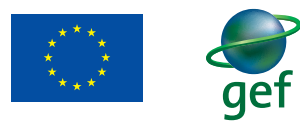

\section{JँIIFAD}

Investing in rural people 